\title{
The rag locus of Porphyromonas gingivalis might arise from Bacteroides via horizontal gene transfer
}

\author{
Z. Su • F. Kong • S. Wang • J. Chen $\cdot$ R. Yin $\cdot$ C. Zhou • \\ Y. Zhang • Z. He $\cdot$ Y. Shi $\cdot$ Y. Xue $\cdot$ X. Shi $\cdot$ L. Lu • \\ Q. Shao $\cdot$ H. Xu
}

Received: 18 November 2009 / Accepted: 5 January 2010/Published online: 2 March 2010

(C) Springer-Verlag 2010

\begin{abstract}
Porphyromonas gingivalis is regarded as one of the risk factors of periodontitis. P. gingivalis exhibits a wide variety of genotypes. Many insertion sequences (ISs), located in their chromosomes, made $P$. gingivalis differentiate into virulent and avirulent strains. In this research, we investigated the prevalence of $P$. gingivalis in the gingival crevicular fluid (GCF) among periodontitis patients from Zhenjiang, China, detected the $P$. gingivalis rag locus distributions by multiplex polymerase chain reaction (PCR), and analyzed the origin of the $P$. gingivalis rag locus based on evolution. There were three rag locus variants co-
\end{abstract}

Z. Su $\cdot$ F. Kong $\cdot$ S. Wang $\cdot$ C. Zhou $\cdot$ Z. He $\cdot$ Y. Shi $\cdot$ Y. Xue $\cdot$

X. Shi $\cdot$ Q. Shao $\cdot$ H. Xu

Department of Immunology and Laboratory Immunology,

Center of Medical Laboratory, Jiangsu University,

Zhenjiang 212013, China

F. Kong $\cdot$ J. Chen $\cdot$ Y. Zhang

The First Affiliated Hospital of Jiangsu University,

Zhenjiang 212005, China

R. Yin

The Affiliated Hospital of Jiangsu University,

Zhenjiang 212001, China

L. $\mathrm{Lu}$

Immunology Division, Department of Pathology,

University of Hong Kong,

Hong Kong, People's Republic of China

S. Wang $(\bowtie) \cdot H$. Xu $(\bowtie)$

School of Medical Science and Laboratory Medicine,

Jiangsu University,

301 Xuefu Road,

Zhenjiang City, Jiangsu Province 212013, China

e-mail: sjwjs@ujs.edu.cn

e-mail: szl30@yeah.net

e-mail: xuhx@ujs.edu.cn existing in Zhenjiang. The results showed that the rag locus may be associated with severe periodontitis. This work also firstly ascertained that the rag locus might arise, in theory, from Bacteroides sp. via horizontal gene transfer.

\section{Introduction}

Porphyromonas gingivalis is a Gram-negative anaerobic bacterium and is considered as a major etiological agent in destructive periodontal diseases [1,2]. It is frequently detected in a high ratio from severely inflamed subgingival lesions, whereas it may also be isolated from the healthy population at a very lower percentage, and these $P$. gingivalis constituted a significant proportion of the oral microflora. Previous studies have also shown phenotypic differences in the pathogenicity of strains of $P$. gingivalis in animal models [3] and in humans [4]. Therefore, P. gingivalis might be an important potential pathogen [5]. Recently, DNA fingerprinting and restriction fragment length polymorphism (RFLP) analysis have confirmed that the highly virulent $P$. gingivalis isolates always carry an rag locus, whereas the avirulent strain does not contain the rag locus [6-8].

The rag locus of virulent $P$. gingivalis encodes $\operatorname{Rag} A$ (receptor antigen gene A), a 115-kDa outer membrane protein with features of a TonB-dependent receptor, and $\operatorname{Rag} B$, a $55-\mathrm{kDa}$ antigen which makes periodontal patients induce an elevated immunoglobulin $\mathrm{G}$ response. The $\operatorname{Rag} A$ and $\operatorname{Rag} B$ form a putative co-distribution and co-transcription complexes on the surface of $P$. gingivalis. The complexes constitute a membrane transporter system, which involves the uptake or recognition of a specific carbohydrate or glycoprotein [9-11]. Four rag locus variants have been detected from periodontal patients [2]. The rag locus has three characteristics: (1) the 
locus has a lower $\mathrm{G}+\mathrm{C}$ content compared with the average values (48\%) of the complete genome; (2) there is an insertion sequence (IS) 1126 with 1,338 bp (IS1126, now known as ISPg1), located upstream of the rag locus and flanked by 12-bp inverted repeats, which is a mobile element that generates a 5-bp target site duplication [12] and contains a single open reading frame (ORF) encoding the transposase which belongs to the IS5 family [13]; (3) polymerase chain reaction (PCR) and Southern blots results indicate that the rag locus has a restricted distribution within the species (only existing in virulent genotypes); the rag locus is also amplified from the subgingival samples of undetected $P$. gingivalis. The characteristics mentioned above make the experts speculate that the rag locus has arisen from exogenous genes and is obtained via horizontal gene transfer, and that the rag locus may represent a pathogenicity island in $P$. gingivalis. However, so far, no report has indicated from where the rag locus arises.

Since from where the rag locus arises is still unknown, the present work was to investigate the prevalence of $P$. gingivalis in the gingival crevicular fluid (GCF) of periodontitis patients from Zhenjiang, China, to detect the rag locus distributions among periodontitis patients infected by $P$. gingivalis using multiplex PCR, and to analyze the origin of the $P$. gingivalis rag locus based on evolution.

\section{Materials and methods}

Subjects, selection of sites, and sampling and DNA extraction

A total of 110 GCF samples were obtained from 110 patients (58 female and 52 male, aged between 15 and 68 years) going to hospital for periodontal diseases at the First Affiliated Hospital of Jiangsu University, China. Patients were screened for periodontal diseases by radiographic evidence of bone loss, or with visible plaque, marginal bleeding, bleeding on probing (BOP), and clinical attachment loss (CAL) and periodontal probing depth $(\mathrm{PD}) \geq 4 \mathrm{~mm}$ at two or more sites
[14, 15]. Exclusion criteria were pregnancy, smoking, and systemic antimicrobial/anti-inflammatory drug therapy within 6 months before the baseline visit. The 110 patients were classified by the 1999 International Workshop for a Classification of Periodontal Diseases and Conditions [16, 17]: 57 patients were localized chronic periodontitis, 33 were generalized periodontitis, and 20 were necrotizing periodontal diseases (three patients were accompanied with diabetes and five patients had cardiovascular disease). The present study was conducted in accordance with the Declaration of Helsinki. All patients gave informed consent and the research protocol was approved by the Committee for Ethical Affairs of Jiangsu University.

GCF samples were obtained from the two deepest periodontal pockets in the maxilla according to Rüdin et al's technique [18]. Before sampling, the respective site was cleaned by cotton rolls; a gentle air stream of 5-s duration with a $90^{\circ}$ angle to the tooth axis was used and supragingival plaque was eliminated. Standardized paper strips were inserted $1 \mathrm{~mm}$ into the sulcus and held for $30 \mathrm{~s}$. Papers with blood contamination were discarded. To avoid evaporation, paper strips with GCF were immediately put into a sterile Eppendorf tube and stored at $-20^{\circ} \mathrm{C}$ until laboratory analysis.

The isolates in the DNA were extracted by the boiling lysis method [19]. The templates were prepared by suspending in $200 \mathrm{~mL}$ of sterile water, followed by boiling for $10 \mathrm{~min}$ and centrifuging for $3 \mathrm{~min}$.

PCR-detected $P$. gingivalis and multiplex PCR amplification of rag locus genes

The 16S rRNA specific primers were used to detect $P$. gingivalis and four different rag locus variants primers were used to amplify the rag locus among GCF samples containing $P$. gingivalis by multiplex PCR; the primers are listed in Table $1[2,20]$. PCR assays were performed in volumes of $15 \mu \mathrm{l}$ under the following conditions: $1.5 \mu \mathrm{l}$ $10 \times$ PCR buffer $\left(\mathrm{Mg}^{+}\right), 0.2 \mathrm{mM}$ of each deoxynucleoside triphosphate (dNTP), $0.1 \mu \mathrm{M}$ of primers, and 1.5 units of
Table 1 Primers used in this study

\begin{tabular}{|c|c|c|c|}
\hline No. & Primers & Sequences & Sizes \\
\hline 1 & $16 S$ rRNAF & TGT AGA TGA CTG ATGGTG AAA ACC & \multirow[t]{2}{*}{$197 \mathrm{bp}$} \\
\hline 2 & $16 S$ rRNAR & ACG TCA TCC CCA CCT TCC TC & \\
\hline 3 & $\operatorname{rag} 1 F$ & CGC GAC CCC GAA GGA AAA GAT T & \multirow[t]{2}{*}{$628 \mathrm{bp}$} \\
\hline 4 & $\operatorname{rag} 1 R$ & CAC GGC TCA CAT AAA GAA CGC T & \\
\hline 5 & $\operatorname{rag} 2 F$ & GCT TTG CCG CTT GTG ACT TGG & \multirow[t]{2}{*}{$979 \mathrm{bp}$} \\
\hline 6 & $\operatorname{rag} 2 R$ & CCA CCGTCA CCG TTC ACC TTG & \\
\hline 7 & $\operatorname{rag} 3 F$ & CCG GAAGAT AAG GCC AAG AAA GA & \multirow[t]{2}{*}{$422 \mathrm{bp}$} \\
\hline 8 & $\operatorname{rag} 3 R$ & ACG CCA ATT CGC CAA AGCT & \\
\hline 9 & $\operatorname{rag} 4 F$ & CCG GAT GGA AGT GAT GAACAG A & \multirow[t]{2}{*}{$738 \mathrm{bp}$} \\
\hline 10 & $\operatorname{rag} 4 R$ & CGC GGT AAA CCT CAG CAA ATT & \\
\hline
\end{tabular}


Taq DNA polymerase (Takara Biotechnology [Dalian] Co., Ltd.) for $10 \mathrm{~min}$ at $94^{\circ} \mathrm{C}$ for 35 cycles, with each cycle consisting of $30 \mathrm{~s}$ at $94^{\circ} \mathrm{C}, 45 \mathrm{~s}$ at $50^{\circ} \mathrm{C}$ (for $16 \mathrm{~S}$ rRNA) and $54^{\circ} \mathrm{C}$ (for the rag locus), and $1 \mathrm{~min}$ at $72^{\circ} \mathrm{C}$, with a final step of $10 \mathrm{~min}$ at $72^{\circ} \mathrm{C}$ for standard PCR assays.

Databases and search strategies

A comprehensive search by PSI-BLAST was performed in the National Center for Biotechnology Information (NCBI) protein database (Reference proteins, RefSeq proteins) using the published $\mathrm{ragAl}$ and $\mathrm{ragBl}$ proteins of $P$. gingivalis as query sequences. The cutoff values were $e-100$ for $\mathrm{rag} A 1$ and $e-10$ for $\mathrm{ragB} 1$, respectively. The redundant sequences were removed by the DAMBE program, and the nucleotide sequences were also retrieved from GenBank.

Additionally, the function-similar proteins of the rag locus were also retrieved from GenBank, and the similar proteins included hemin/hemoglobin receptor protein (HmuR), TonB-linked adhesin (Tla) of P. gingivalis, TonB-dependent colicin I receptor (CirA) of Escherichia coli and Salmonella enterica $[9,21]$, and the lactoferrin and transferrin binding systems in Neisseria and Haemophilus species (TBP2 and LBP2), P. gingivalis orf3, Vibrio cholerae-IS1358, and E. coli orfH. These similar proteins were used to analyze the gene characteristics.

Sequence comparison and phylogenetic analysis

Multiple sequence alignments were performed using the ClustalX package, and then the results were manually corrected. All alignment gap sites were eliminated before phylogenetic analyses. The phylogenetic trees were reconstructed with the neighbor-joining (NJ) and minimum evolution (ME) methods implemented in MEGA4.0. The bootstrap values were estimated with 1,000 replications. Multiple sequence alignments were also carried out between the rag locus and highly homologous sequences by ClustalX in order to further identify conserved protein motifs. The alignments were shaded by the BOXSHADE program (http://www.ch.embnet.org/software/BOX_form.html).

Tests for selection

To test whether the rag locus was evolving neutrally, we aligned the nucleotide sequences and then performed two likelihood ratio tests. Firstly, we compared a model in which all branches had the same $\mathrm{dN} / \mathrm{dS}$ ratio of 1 , in which each branch had its own ratio, to determine whether it was appropriate to use branch-specific tests for selection. As the free-ratio model was a significantly better fit to the data $(P>0.0001)$, we then performed a second set of tests, comparing a model in which the dN/dS ratio was fixed to 1 with a model in which the $\mathrm{dN} / \mathrm{dS}$ values of all branches were freely estimated. Model likelihoods were estimated using PAML [22].

\section{Results}

The prevalence of $P$. gingivalis and four rag locus variant gene distributions

We detected 29 P. gingivalis from $110 \mathrm{GCF}$ samples, one from 57 cases of localized chronic periodontitis, nine from 33 generalized chronic periodontitis patients, and 19 from 20 necrotizing periodontal patients. Among the $29 \mathrm{GCF}$ samples containing $P$. gingivalis, only 19 cases were amplified from the rag locus genes (ten to ragl, six to rag2, and three to rag3), while rag4 was undetected. Among the 19 cases containing the rag locus, 18 cases were amplified from necrotizing periodontal patients and one from a generalized chronic periodontitis patient. In particular, three patients were accompanied with diabetes and five patients who suffered from cardiovascular diseases also amplified the rag locus, and all of the 19 cases were from at least three sites with a PD of 8 to $10 \mathrm{~mm}$, accompanying clearly visible plaque or $\mathrm{BOP}$ and $\mathrm{CAL}$, whereas none of the localized chronic periodontitis patients amplified the rag locus and the PD of the localized chronic periodontitis patients was normally between $4-6 \mathrm{~mm}$ (Fig. 1).

\section{Phylogenetic analysis}

A total of 153 homologous amino acid sequences were retrieved from the RefSeq proteins. The abundant sequences were removed by the DAMBE program and a total 50 protein sequences were included in the final dataset. In order to determine the evolutionary relationships between the $P$. gingivalis rag locus and homologous sequences, the phylogenetic trees were reconstructed by two different methods (ME and NJ methods) with high bootstrap values. There were three major clusters (I, II, and III), which were statistically supported (Fig. 2). Cluster I mainly included Bacteroides sp. TonB-dependent receptor (SusC/CirA), P. gingivalis ragA, Porphyromonas $\mathrm{sp}$ ragA, and Capnocytophaga sp. TonB-dependent receptor; cluster II was constituted of TonB-dependent receptor of Parabacteroides sp., Dyadobacter fermentans, Robiginitalea biformata, Capnocytophaga ochracea, Flavobacterium johnsoniae, Croceibacter atlanticus, Bacteroides sp., Dyadobacter fermentans, and Parabacteroides sp.; and P. gingivalis ragB, Porphyromonas sp. ragB and SusD of Prevotella sp., Bacteroides sp., and Capnocytophaga sp. were involved in the cluster III. 
Fig. 1a-c The prevalence of Porphyromonas gingivalis and four rag locus variant gene distributions. a Agarose gel electrophoresis image of $P$. gingivalis detection. Lane 1 marker, lane 2 positive control, lane 11 negative control, lanes 3-10 samples. b Image of four rag locus variants gene distributions. Lane 1 marker, lane 2 positive control, lane 11 negative control, lanes 3-10 were samples. c Case numbers of varying degrees of periodontitis, the number of $P$. gingivalis detected in different periodontitis patients, and the four rag locus variant gene distributions a

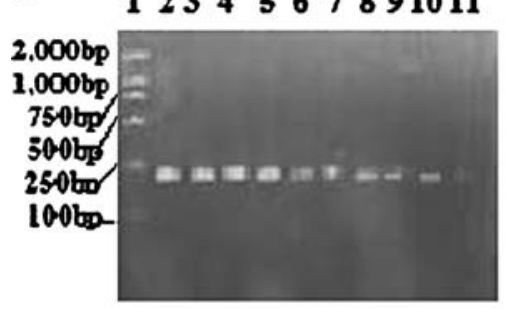

c

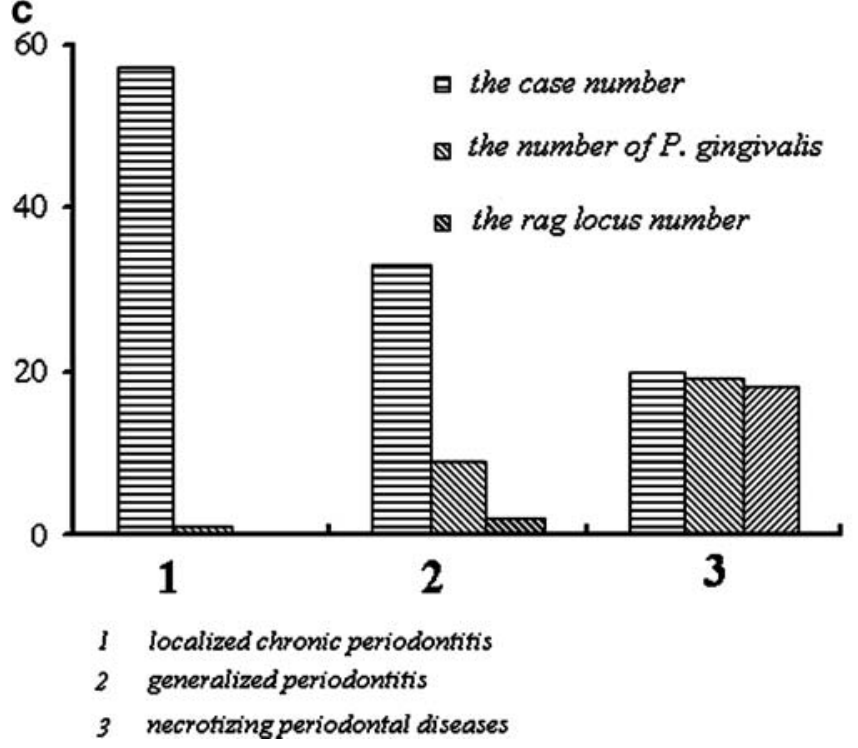

12344567891011

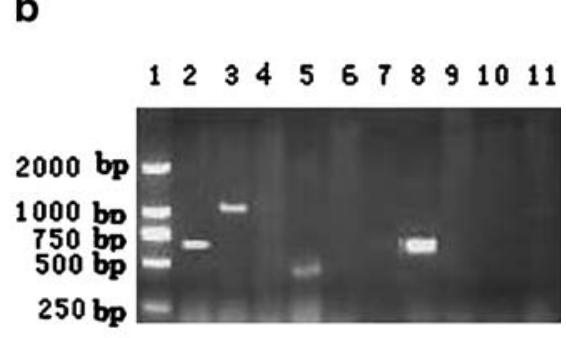

$2000 \mathrm{~b}$

50 bo

00 bp

$50 \mathrm{bp}$ 
Fig. 2 Phylogenetic relationship between the $P$. gingivalis $\mathrm{rag} A / B$ and highly homologous protein sequences

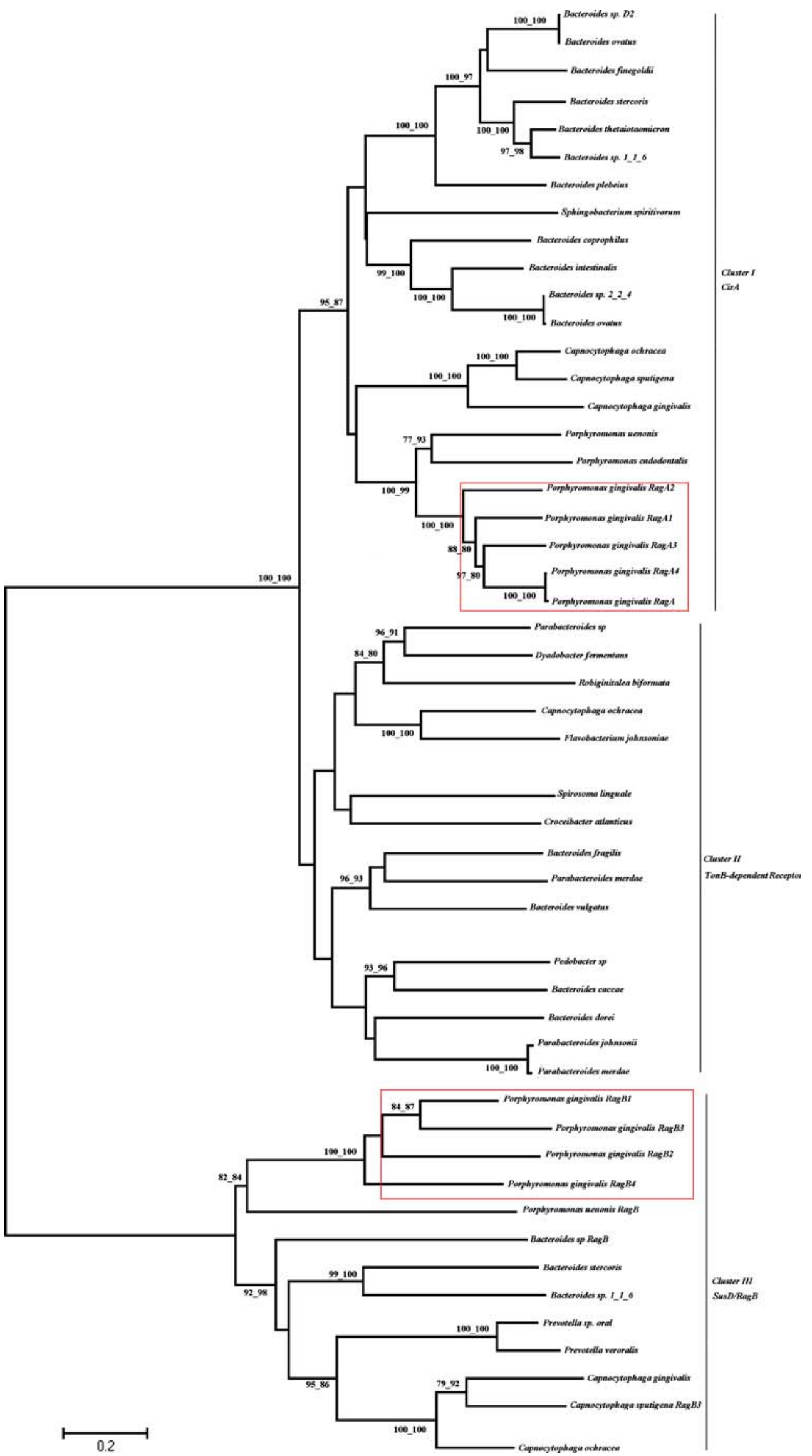


Table $2 \mathrm{G}+\mathrm{C}$ contents of the rag locus, homologous genes, and function-similar sequences

\begin{tabular}{|c|c|c|c|c|c|c|}
\hline Strains & Encode protein & $\mathrm{T}(\%)$ & $\mathrm{C}(\%)$ & A $(\%)$ & $\mathrm{G}(\%)$ & $\mathrm{G}+\mathrm{C}(\%)$ \\
\hline Flavobacterium sp. & TonB-dependent receptor & 31.7 & 16.4 & 34.9 & 16.9 & 33.3 \\
\hline Croceibacter sp. & TonB & 35.1 & 20.4 & 29.2 & 15.3 & 35.7 \\
\hline H. influenza & $T B P 2$ & 36.3 & 18.5 & 27.4 & 17.8 & 36.3 \\
\hline Haemophilus & TonB-dependent receptor & 34.4 & 20 & 27.4 & 18.2 & 38.2 \\
\hline Capnocytophaga sp. & TonB-dependent receptor & 31.3 & 20.2 & 28.8 & 20.7 & 40.9 \\
\hline E. coli & orfH & 25.8 & 17.6 & 32.5 & 24.2 & 41.8 \\
\hline V. cholerae & IS1358 & 27.9 & 17.9 & 29.9 & 24.3 & 42.2 \\
\hline P. gingivalis & $\operatorname{orf3}$ & 31 & 20.8 & 25.8 & 22.3 & 43.1 \\
\hline P. gingivalis & $\operatorname{Rag} B$ & 29.4 & 19.5 & 27 & 23.9 & 43.4 \\
\hline Bacteroides sp. & SusC & 28.0 & 23.5 & 26.5 & 22.0 & 43.5 \\
\hline$P$. gingivalis & $\operatorname{Rag} A$ & 29 & 20.5 & 26.7 & 23.9 & 44.4 \\
\hline Parabacteroides & TonB-dependent receptor & 27 & 19.8 & 26.9 & 26.3 & 46.1 \\
\hline N. meningitidis & $L B P 2$ & 31.5 & 25.4 & 22.1 & 20.9 & 46.3 \\
\hline P. gingivalis & hmuR & 26.2 & 24.8 & 27.2 & 21.9 & 46.7 \\
\hline N. meningitidis & $T B P 2$ & 20.2 & 23 & 32.4 & 24.4 & 47.4 \\
\hline$P$. gingivalis & hemR & 26.8 & 25.2 & 25.8 & 22.2 & 47.4 \\
\hline$P$. gingivalis & tla & 24.2 & 24.6 & 26.7 & 24.4 & 49 \\
\hline E. coli & CirA & 21.5 & 24.9 & 26.2 & 27.3 & 52.2 \\
\hline Robiginitalea sp. & $\operatorname{Ton} B$ & 26.7 & 29.6 & 20.1 & 23.5 & 53.1 \\
\hline Salmonella enterica & CirA & 20.8 & 25.8 & 24.6 & 28.8 & 54.6 \\
\hline
\end{tabular}

it is definite that $P$. gingivalis has been known to be a risk factor for periodontitis. Furthermore, accumulated evidence shows that an infection with $P$. gingivalis might predispose an individual to suffer from cardiovascular disease, diabetes, and the delivery of preterm infants [2326]. All of these factors highlighted the importance of preventing infection from this bacterium. In this respect, information on the virulent factors and genotype of $P$. gingivalis among individuals becomes important for developing treatment strategies and preventing the transmission of the pathogen to uninfected individuals [12]. Previous researches stated that $P$. gingivalis produced a number of well-characterized virulence factors, including proteases, fimbriae, and capsule [27]. Recently, a significant association was observed between a carrier of the rag locus and a highly virulent phenotype in a murine model of soft tissue destruction [3, 4, 10, 28].

In order to further determine whether the $P$. gingivalis rag locus was associated with periodontitis, we investigated the prevalence of $P$. gingivalis and rag locus distributions in periodontitis patients in this study. We detected $29 P$. gingivalis out of 110 patient GCF samples. However, the prevalence of $P$. gingivalis was lower in localized chronic periodontitis patients $(1.75 \%)$ and generalized chronic periodontitis patients (27\%) [29]. We also suspected that the method of samples collection was insensitive, but others reported that the prevalence of $P$. gingivalis was high by this method [30, 31]. At the same time, the ratios of
P. gingivalis in necrotizing periodontal patients (95\%) were in accordance with other reports [32-34]. Among the 29 cases containing $P$. gingivalis, only 19 samples amplified the rag locus variants. And among 19 cases containing the rag locus, 18 (95\%) were amplified from necrotizing periodontal patients and one (5\%) from a generalized chronic periodontitis patient. All of the 19 cases containing the rag locus were from at least three sites with a PD of 8 to $10 \mathrm{~mm}$, accompanying clearly visible plaque or BOP and CAL; at the same time, some rag locus carrier cases accompanied diabetes and cardiovascular disease, which indicated that the rag locus may be associated with severe periodontitis. Besides, we found that ragl locus variants may be the prevalent genotype in Zhenjiang; however, other rag locus variants were also common in other regions [29, 34-37], which showed that the rag locus distribution had the same obvious regional differences as the bacteria resistance to antibiotics [38].

The rag locus has a restricted distribution within the species and lower $\mathrm{G}+\mathrm{C}$ contents. Furthermore, the rag locus is located downstream of IS1126. As was well known that the insertion sequence (IS) is a mobile element contributing to gene transposition which could lead to the inactivation of genes, the transcriptional activation of dormant genes, and genomic rearrangements, which are the main causes of the pathogenicity and genetic diversity of bacterial populations [39-41], we speculated that the rag locus might have arisen from exogenous genes and 


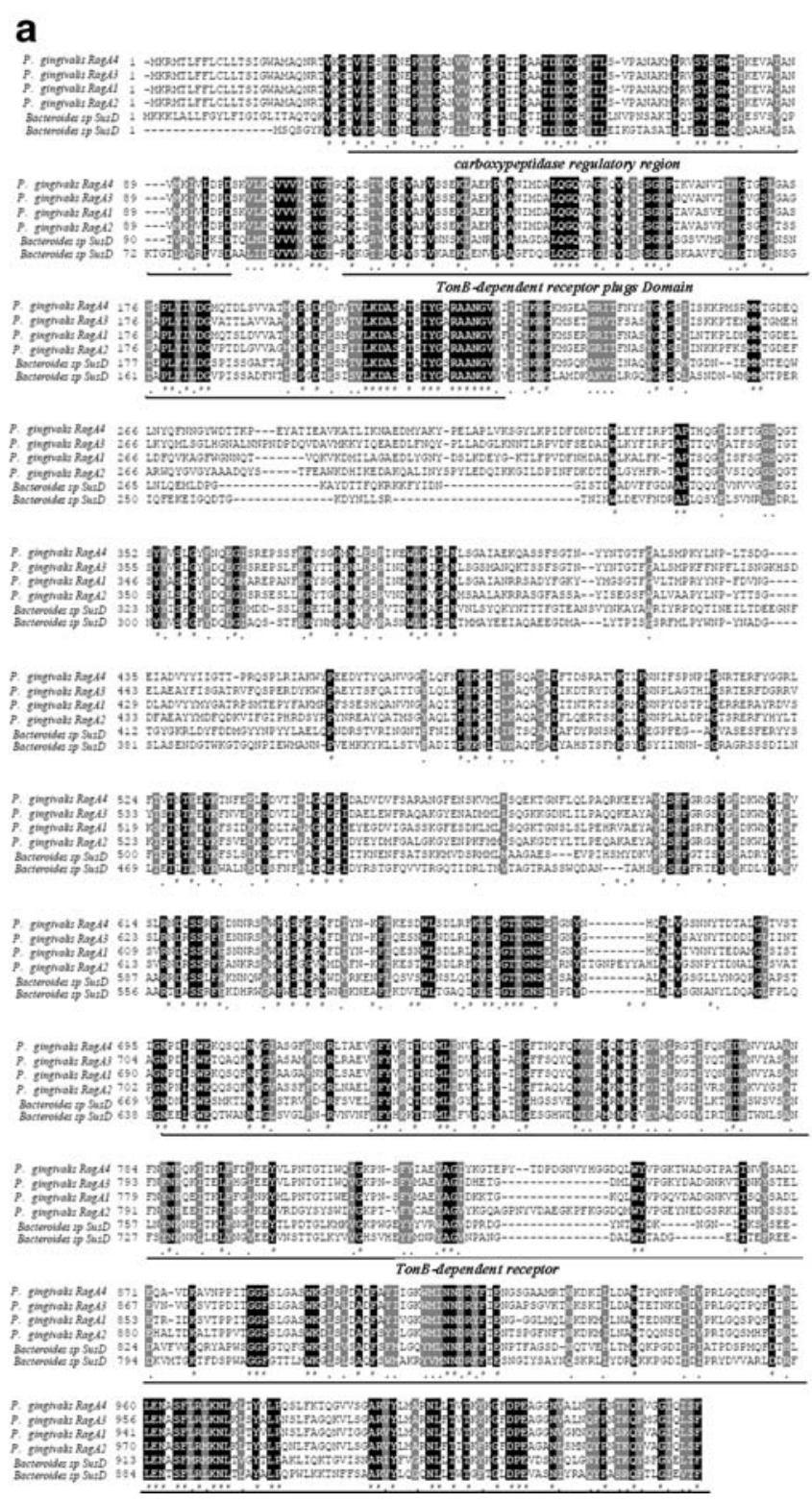

Fig. 3a, b Comparison of the $P$. gingivalis rag locus with Bacteroides Sus. a, b Multiple amino acid sequences alignment between the $P$. gingivalis $\operatorname{Rag} A$ locus with Bacteroides SusC. The asterisks indicate

obtained through transposition. In order to prove our speculations, a phylogenetic analysis was performed to determine the evolutionary relationships between the $P$. gingivalis rag locus and homologous sequences. Many proteins were analyzed, for example, RagA/B, Sus, and CirA. All of these proteins belonged to the family of TonBlinked outer membrane receptors which were involved in the recognition and active transport of specific external ligands [36]. In organisms, a TonB-linked outer membrane receptor was also co-transcribed with an outer membrane lipoprotein [42]. The co-distribution and co-transcription of $\operatorname{rag} A$ and $\operatorname{rag} B$ strongly suggested that they were function-

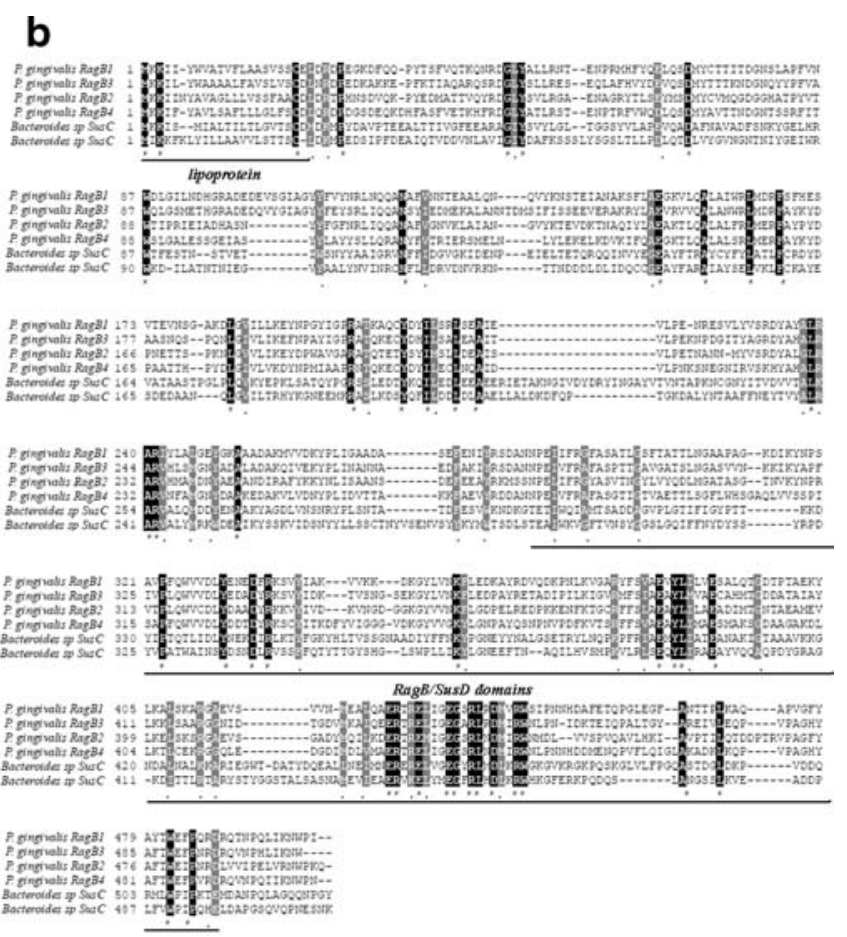

positions within the proteins that were identical. The carboxypeptidase regulatory region, TonB-dependent receptor plug Domain, TonBdependent receptor, lipoprotein, and $\operatorname{RagB} / \mathrm{Sus} D$ domains are shown

ally linked, and the products might form a complex on the outer surface of $P$. gingivalis, which was involved in a TonB-linked process. RagA, Sus, and CirA were involved in maltose uptake, and $\operatorname{Rag} B$ was associated with the active transport of lactoferrin and transferrin iron sources. $\operatorname{Rag} B$ was closely similar to $\operatorname{RagB} / S u s D$ of Prevotella sp., Bacteroides sp., and Capnocytophaga sp. Furthermore, this tree also showed that branches 1 and 2 in cluster I were the paralogy, and in cluster III, the two branches were also paralogy, which indicated that $\operatorname{rag} A$ was highly similar to the TonB-dependent receptor of Bacteroides sp., and $\operatorname{rag} B$ was analogous to $\mathrm{ragB} / \mathrm{SusD}$ of Prevotella sp., Bacteroides 
sp., and Capnocytophaga sp., respectively. To further determine the origin of the rag locus, the $\mathrm{G}+\mathrm{C}$ contents of highly homologous genes to rag were analyzed. The analysis of $\mathrm{G}+\mathrm{C}$ contents revealed that the $\mathrm{G}+\mathrm{C}$ contents of the rag locus were under the average level of the $P$. gingivalis complete genome, including $H m u R$, and that the $\mathrm{G}+\mathrm{C}$ contents of the rag locus were close to Bacteroides sp. Sus. Moreover, multiple alignments between the rag locus and Sus showed that the $\operatorname{RagA}$ and $\operatorname{SusC}$ were both constituted of three domains (TonB I, II, and III); and $\operatorname{Rag} B$ and $\operatorname{Sus} D$ were constituted of two domains: Lipoprotein and $\operatorname{Rag} B / S u s D$, respectively. The amino acids were highly conserved, and our tests for selection showed that the rag locus were probably evolving under long-term purifying selection, which also indirectly indicated that the rag locus might arise from horizontal gene transfer.

From all of the evidence mentioned above, we could conclude that the rag locus was not duplicated by self$H m u R$, but instead arose from Bacteroides sp. Sus via horizontal gene transfer. The exogenous rag locus promoted the $P$. gingivalis expression of virulence gene or the activation of dormant genes. Thus, $P$. gingivalis was differentiated into virulent and avirulent genotype. The rag locus-carried genotype may be associated with severe periodontitis.

Acknowledgments This work was supported by the social development of Jiangsu province (Grant No. BS2007041), the SCI-Tech Innovation Team of Jiangsu University (Grant No. 2008-018-02), the National Natural Science Foundation of China (Grant No. 30871193), the Natural Science Foundation of Colleges and Universities in Jiangsu Province, and the Innovation Fund for candidate of doctor in Jiangsu Province (Grant Nos. 09KJB310001 and CX09B_217Z, respectively), and the High-Level Creative Talent Development Project of Jiangsu Province (07-D-034).

We thank Yu Zhang, Hui Cao, Daoru Dong, Dr. F. Valiyeva, and $\mathrm{Dr}$. Hu $\mathrm{Xu}$ for their editing and proofreading of the manuscript.

\section{References}

1. Griffen AL, Becker MR, Lyons SR, Moeschberger ML, Leys EJ (1998) Prevalence of Porphyromonas gingivalis and periodontal health status. J Clin Microbiol 36(11):3239-3242

2. Hall LM, Fawell SC, Shi X, Faray-Kele MC, Aduse-Opoku J, Whiley RA, Curtis MA (2005) Sequence diversity and antigenic variation at the rag locus of Porphyromonas gingivalis. Infect Immun 73(7):4253-4262

3. Genco CA, Cutler CW, Kapczynski D, Maloney K, Arnold RR (1991) A novel mouse model to study the virulence of and host response to Porphyromonas (Bacteroides) gingivalis. Infect Immun 59(4): 1255-1263

4. Griffen AL, Lyons SR, Becker MR, Moeschberger ML, Leys EJ (1999) Porphyromonas gingivalis strain variability and periodontitis. J Clin Microbiol 37(12):4028-4033

5. Rylev M, Kilian M (2008) Prevalence and distribution of principal periodontal pathogens worldwide. J Clin Periodontol $35(8$ Suppl):346-361
6. Loos BG, Mayrand D, Genco RJ, Dickinson DP (1990) Genetic heterogeneity of Porphyromonas (Bacteroides) gingivalis by genomic DNA fingerprinting. J Dent Res 69(8):1488-1493

7. Genco RJ, Loos BG (1991) The use of genomic DNA fingerprinting in studies of the epidemiology of bacteria in periodontitis. J Clin Periodontol 18(6):396-405

8. Zhang YJ, Yasui S, Yoshimura F, Ishikawa I (1995) Multiple restriction fragment length polymorphism genotypes of Porphyromonas gingivalis in single periodontal pockets. Oral Microbiol Immunol 10(2):125-128

9. Hanley SA, Aduse-Opoku J, Curtis MA (1999) A 55-kilodalton immunodominant antigen of Porphyromonas gingivalis W50 has arisen via horizontal gene transfer. Infect Immun 67(3):1157-1171

10. Curtis MA, Hanley SA, Aduse-Opoku J (1999) The rag locus of Porphyromonas gingivalis: a novel pathogenicity island. J Periodontal Res 34(7):400-405

11. Curtis MA, Slaney JM, Carman RJ, Johnson NW (1991) Identification of the major surface protein antigens of Porphyromonas gingivalis using IgG antibody reactivity of periodontal case-control serum. Oral Microbiol Immunol 6(6):321-326

12. Park OJ, Min KM, Choe SJ, Choi BK, Kim KK (2004) Use of insertion sequence element IS1126 in a genotyping and transmission study of Porphyromonas gingivalis. J Clin Microbiol 42 (2):535-541

13. Maley J, Roberts IS (1994) Characterisation of IS1126 from Porphyromonas gingivalis W83: a new member of the IS4 family of insertion sequence elements. FEMS Microbiol Lett 123(1-2):219224

14. Airila-Månsson S, Söder B, Kari K, Meurman JH (2006) Influence of combinations of bacteria on the levels of prostaglandin E2, interleukin-1beta, and granulocyte elastase in gingival crevicular fluid and on the severity of periodontal disease. J Periodontol 77 (6):1025-1031

15. Gomes SC, Piccinin FB, Oppermann RV, Susin C, Nonnenmacher CI, Mutters R, Marcantonio RA (2006) Periodontal status in smokers and never-smokers: clinical findings and real-time polymerase chain reaction quantification of putative periodontal pathogens. J Periodontol 77(9):1483-1490

16. Armitage GC (2000) Development of a classification system for periodontal diseases and conditions. Northwest Dent 79(6):31-35

17. Armitage GC (1999) Development of a classification system for periodontal diseases and conditions. Ann Periodontol 4(1):1-6

18. Rüdin HJ, Overdiek HF, Rateitschak KH (1970) Correlation between sulcus fluid rate and clinical and histological inflammation of the marginal gingiva. Helv Odontol Acta 14(1):21-26

19. Lévesque C, Piché L, Larose C, Roy PH (1995) PCR mapping of integrons reveals several novel combinations of resistance genes. Antimicrob Agents Chemother 39(1):185-191

20. Tran SD, Rudney JD (1996) Multiplex PCR using conserved and species-specific 16S rRNA gene primers for simultaneous detection of Actinobacillus actinomycetemcomitans and Porphyromonas gingivalis. J Clin Microbiol 34(11):2674-2678

21. Chen T, Hosogi Y, Nishikawa K, Abbey K, Fleischmann RD, Walling J, Duncan MJ (2004) Comparative whole-genome analysis of virulent and avirulent strains of Porphyromonas gingivalis. J Bacteriol 186(16):5473-5479

22. Yang Z (1997) PAML: a program package for phylogenetic analysis by maximum likelihood. Comput Appl Biosci 13(5):555556

23. Persson GR, Imfeld $T$ (2008) Periodontitis and cardiovascular disease. Ther Umsch 65(2):121-126

24. Seymour GJ, Ford PJ, Cullinan MP, Leishman S, Yamazaki K (2007) Relationship between periodontal infections and systemic disease. Clin Microbiol Infect 13(Suppl 4):3-10

25. Offenbacher S, Jared HL, O'Reilly PG, Wells SR, Salvi GE, Lawrence HP, Socransky SS, Beck JD (1998) Potential pathogenic 
mechanisms of periodontitis associated pregnancy complications. Ann Periodontol 3(1):233-250

26. Campus G, Salem A, Uzzau S, Baldoni E, Tonolo G (2005) Diabetes and periodontal disease: a case-control study. J Periodontol 76(3):418-425

27. Holt SC, Kesavalu L, Walker S, Genco CA (1999) Virulence factors of Porphyromonas gingivalis. Periodontol 2000 20:168-238

28. Shi X, Hanley SA, Faray-Kele MC, Fawell SC, Aduse-Opoku J, Whiley RA, Curtis MA, Hall LM (2007) The rag locus of Porphyromonas gingivalis contributes to virulence in a murine model of soft tissue destruction. Infect Immun 75(4):2071-2074

29. Siqueira JF Jr, Rôças IN, Silva MG (2008) Prevalence and clonal analysis of Porphyromonas gingivalis in primary endodontic infections. J Endod 34(11):1332-1336

30. Darby IB, Mooney J, Kinane DF (2001) Changes in subgingival microflora and humoral immune response following periodontal therapy. J Clin Periodontol 28(8):796-805

31. Ling LJ, Hung SL, Tseng SC, Chen YT, Chi LY, Wu KM, Lai YL (2001) Association between betel quid chewing, periodontal status and periodontal pathogens. Oral Microbiol Immunol 16(6):364-369

32. Riep B, Edesi-Neuss L, Claessen F, Skarabis H, Ehmke B, Flemmig TF, Bernimoulin JP, Göbel UB, Moter A (2009) Are putative periodontal pathogens reliable diagnostic markers? J Clin Microbiol 47(6):1705-1711

33. Torrungruang K, Bandhaya P, Likittanasombat K, Grittayaphong C (2009) Relationship between the presence of certain bacterial pathogens and periodontal status of urban Thai adults. J Periodontol 80(1):122-129

34. Faghri J, Moghim Sh, Abed AM, Rezaei F, Chalabi M (2007) Prevalence of Porphyromonas gingivalis and Bacteroides for- sythus in chronic periodontitis by multiplex PCR. Pak J Biol Sci 10(22):4123-4127

35. Kulekci G, Leblebicioglu B, Keskin F, Ciftci S, Badur S (2008) Salivary detection of periodontopathic bacteria in periodontally healthy children. Anaerobe 14(1):49-54

36. Sakai VT, Campos MR, Machado MA, Lauris JR, Greene AS, Santos CF (2007) Prevalence of four putative periodontopathic bacteria in saliva of a group of Brazilian children with mixed dentition: 1-year longitudinal study. Int J Paediatr Dent 17 (3):192-199

37. Koebnik R (2005) TonB-dependent trans-envelope signalling: the exception or the rule? Trends Microbiol 13(8):343-347

38. Su Z, Xu H, Zhang C, Shao S, Li L, Wang H, Qiu G (2006) Mutations in Helicobacter pylori porD and oorD genes may contribute to furazolidone resistance. Croat Med J 47(3):410-415

39. Podglajen I, Breuil J, Casin I, Collatz E (1995) Genotypic identification of two groups within the species Bacteroides fragilis by ribotyping and by analysis of PCR-generated fragment patterns and insertion sequence content. J Bacteriol 177(18):5270-5275

40. Bik EM, Gouw RD, Mooi FR (1996) DNA fingerprinting of Vibrio cholerae strains with a novel insertion sequence element: a tool to identify epidemic strains. J Clin Microbiol 34(6):14531461

41. Guerrero C, Bernasconi C, Burki D, Bodmer T, Telenti A (1995) A novel insertion element from Mycobacterium avium, IS1245, is a specific target for analysis of strain relatedness. J Clin Microbiol 33(2):304-307

42. Cornelissen CN (2003) Transferrin-iron uptake by Gram-negative bacteria. Front Biosci 8:d836-d847 